Neurosurg Focus 12 (3):Article 4, 2002, Click here to return to Table of Contents

\title{
Weapons of mass destruction: radiation
}

\author{
Chris J. Neal, M.D., ANd Leon E. Moores, M.D. \\ Pediatric Neurosurgery, National Capital Consortium, Walter Reed Army Medical Center, \\ Washington, D.C.
}

\begin{abstract}
The purpose of this review is to present a concise overview of the types of radiation, methods of dispersal, injury patterns, and treatment considerations in a scenario involving radiation-based weapons of mass destruction. Radiationrelated casualties, although uncommon, are a potential threat because more nations and organizations are developing the technology for producing radioactive substances capable of being used as weapons.
\end{abstract}

KEY WORDS • radiation • nuclear warfare • casualties • war

The perception about radioactive substances has changed over the last century. Prior to the detonations of nuclear bombs in Hiroshima and Nagasaki, radioactive substances were used in a variety of public sources, such as luminous paints, with minimal concern for their pathological effects. Public sensitivity continued to change during the Cold War, as fear of nuclear warfare mounted. Current availability of radioactive substances and technology, as well as the threat of terrorist use, have raised threat credibility to levels not seen since the end of the Cold War.

\section{WEAPONS OF MASS DESTRUCTION: RADIATION}

Radiation-induced casualties can be caused by a variety of devices. Nuclear weapons, although a great public concern, are not the most likely source of radiation injury. Limited availability of weapons-grade fuel, tremendous technical obstacles, complexity of delivery, and expense of maintenance limit proliferation. A more probable threat is a "dirty bomb," a device constructed to disseminate radioactive material without a nuclear detonation. ${ }^{1}$ Radioactive material for such a bomb can be obtained from innocuous sources including university research facilities, medical radiotherapy clinics, industrial complexes, nuclear power plants, and nuclear waste processors. A similar effect can be achieved by destroying a target that contains radioactive elements. A hijacked truck carrying radioactive waste, for example, can be used for dispersal of radioactive elements without an explosion to contaminate a public water system or space.

Abbreviations used in this paper: $\mathrm{GI}=$ gastrointestinal; $\mathrm{US}=$ United States.
The terminology used to describe any dose of radiation conforms to the International System of Units for radiation absorbed dose, the gray. This energy equals 1 joule per kilogram, or $100 \mathrm{Rad}$. Alpha particles, beta particles, gamma rays, and neutrons are four types of clinically relevant radiation. Alpha particles are large, charged particles that can travel short distances. Although stopped by clothing, when alpha particles are internalized, they can cause significant localized damage. Beta particles are light, charged particles found in fallout radiation, which travel a short distance in tissue. They produce dose-dependent damage to the basal stratum of the skin similar to thermal injury (a "beta burn"). Gamma rays are uncharged, highly energetic radiation that thoroughly penetrate all body tissue. Neutrons are uncharged particles only emitted during a nuclear detonation. Because of their size, they interrupt atomic structures and cause 20 -fold greater damage than gamma radiation. ${ }^{1}$

Radiation-induced illness can be acute or chronic, depending on the dose and duration of exposure. Other damage includes carcinogenesis, infertility, and fetal effects. The $\mathrm{LD}_{50 / 60}$, the median lethal dose of radiation that will kill $50 \%$ of the exposed persons within 60 days, is 3.5 Gy when medical care is not instituted. ${ }^{1}$ Recovery of an organ system exposed to such an insult will depend on the damage to the stem cell population, with delayed somatic effects being common.

Acute radiation syndrome has variable symptoms depending on the radiation dose, type, and individual sensitivity. The prodromal phase is marked by rapid onset of nausea, vomiting, and malaise. The latent phase follows and is relatively symptom free.

Individuals exposed to 0.7 to $4 \mathrm{~Gy}$ will suffer bone marrow effects, with changes in the peripheral blood smear 


\section{J. Neal and L. E. Moores}

seen as early as 24 hours postexposure. Bone marrow suppression causes an increased risk of infection and anemia from 10 to 60 days postirradiation. In acute radiation syndrome, prevention and management of infection is critical. The extent of neutropenia is the greatest risk factor for infection. The management of a patient in whom neutropenia occurs secondary to radiation exposure is no different from cases of neutropenia from other causes. ${ }^{1}$ In a conventional nuclear detonation, the majority of victims who reach medical care will fall into this categorypatients exposed to higher doses die instantly or imminently of thermal- or blast-related injuries. In nonconventional radiation dispersal scenarios, however, higher-dose exposures may occur in survivors. In either situation, lowdose exposure produces injury that can be treated.

Exposure to 6 to 8 Gy of radiation results in a GI syndrome. Hemorrhage, fluid loss, and diarrhea are common manifestations of the GI syndrome as the mucosa is denuded of functional cells after villi radiation damage. With concomitant injury to the microvasculature, shock can occur within 1 to 2 weeks. ${ }^{1}$

A neurovascular syndrome occurs in victims exposed to acute high-dose radiation of 20 to $40 \mathrm{~Gy}$. In these cases the latent phase lasts only hours before the onset of symptoms. Clinically, these patients deteriorate into a coma resulting in death. Convulsions may or may not occur with few signs or symptoms to suggest an increased intracranial pressure. Exposure to this amount of radiation in a nuclear explosion would cause $100 \%$ lethality due to blast and thermal effects. With this level of exposure, survival is not likely. ${ }^{1}$ In the event of a mass-casualty situation for which limited resources are available, patients should be sorted in triage to the category of "expectant" if they exhibit mental status changes attributable to radiation exposure.

Chronic radiation syndrome occurs as a result of longterm radiation exposure. Patients present with neuroregulatory disorders, leukopenia, and thrombocytopenia. In severe cases anemia, atrophy of the GI membranes, and encephalomyelitis can be observed. Exposure to at least 1 Gy/year for 3 years may result in symptoms. ${ }^{1}$ Patients present with localized tenderness, hypotension, tachycardia, intention tremor, ataxia, asthenia, or hyperreflexia. Removal of the patient from the source of radiation may resolve the symptoms.

Radiation exposure may result in carcinogenesis. The annual lifetime risk in the US of a fatal cancer is $20 \%$. An exposure to $100 \mathrm{mGy}$ causes a $0.8 \%$ increase in the lifetime risk of cancer-related death. This exposure is double the US occupational annual limit. The period between exposure and carcinogenesis is unpredictable but may be as long as decades. ${ }^{1}$

Radiation adversely affects fertility. Sublethal doses of radiation result in a transient period of azoospermia with sterility lasting months to years. Germ cells, when affected, do not produce mature sperm or ova and therefore do not transmit genetic abnormalities that could occur from the radiation. Theoretically, radiation exposure increases the rate of point mutations and thus could result in an increase in genetic abnormalities in subsequent generations. This has not been seen in humans. ${ }^{1}$

Fetal exposure to radiation has three main effects: death, teratogenesis, and developmental abnormalities.
Death occurs if sufficient irradiation occurs prior to implantation. Teratogenesis occurs during organogenesis, and the central nervous system is often affected. Analysis of data obtained from survivors of Hiroshima and $\mathrm{Na}-$ gasaki showed that congenital microcephaly could result from free-in-air radiation doses of 100 to $190 \mathrm{mGy} .{ }^{1} \mathrm{De}-$ velopmental abnormalities and growth retardation have been noted following exposure in the fetal period.

The detonation of a nuclear weapon results in two types of blast forces and two types of thermal injury. The first blast force results from direct overpressurization. This does not extend far from the point of detonation, and the extent of the damage depends on the rate of pressure change at the blast wave front, the magnitude of the peak overpressurization, and the duration of the blast wave. ${ }^{2}$ The more clinically significant indirect wind drag force is proportional to the velocity and duration of blast winds. These tremendous energies vary from the point of detonation, yield of the weapon, and altitude of the burst, and are of short duration. Injuries occur as mobile objects become missiles and as individuals are blown into stable structures. $^{2}$

Thermal injuries result from the direct absorption of thermal energy (flash burns) and/or indirect environment fires (flame burns). The pattern of flash burns depends on the patient's type of clothing and coverage. Exposed skin facing the blast will be burned, sparing the contralateral side. The amount of energy absorbed by the clothingcovered skin depends on the properties of the clothes. Dark-color clothing absorbs infrared energy, resulting in pattern burns, whereas light colors reflect this energy. Secondary fires result in common flame burns that may be complicated by inhalation injury. ${ }^{2}$

When treating an injured contaminated patient in an acute setting, the provider should realize that it is highly unlikely that a survivor is so contaminated that he poses a radiation hazard to the provider. ${ }^{1}$ Initial decontamination steps should be performed outside the medical facility. Confirmation of radiation contamination is performed by use of a radiation detector (a radiac). Prior to decontamination, cover open wounds and remove contaminated clothing, placing clothing in labeled plastic bags that will be discarded. Removing clothing and washing exposed skin and hair rids the victim of approximately 95\% of the contaminant. ${ }^{1}$ Hypochlorite solution $(0.5 \%)$ can be used to wash the body. The skin must not be irritated because this increases the absorption of some radioactive particles. Wash solution should be collected and disposed of appropriately. If this is not feasible, flush water to standard drains and notify the local water treatment facility.

Remove all bandages and flush wounds liberally. Remove particulate matter in the wound and debride. A radiac is used to determine adequate wound decontamination. All fluids must be evacuated from the wound because they may mask the detection of alpha and beta particles. Suction, rather than sponging should be used to remove fluids to decrease environmental contamination. After the wound is decontaminated, it should be flushed again. Do not perform aggressive surgical procedures to remove trace contamination, because the lifetime risk from radiation exposure is less than the risk of immediate wound complications and infection. 


\section{Weapons of mass destruction: radiation}

Treatment of acute or life-threatening injury must precede treatment for radiation injury. Immune system compromise and delayed wound healing, however, may compromise any treatment provided. Surgical procedures should be performed within 48 hours of the radiation injury, and sooner is better. Wound sites should be closed primarily because wound healing is markedly compromised within hours of radiation injury. ${ }^{1}$ Anticontamination coveralls should be worn by the provider prior to the patient's initial decontamination, but standard universal precautions are adequate for those treating limited numbers of radiologically contaminated patients. After treating and decontaminating the patient, providers themselves should undergo decontamination.

Although it is difficult to predict the method of delivery of a radiation threat, common diagnostic and treatment algorithms apply based on the acuity, radiation dose, and duration of exposure. Physicians, particularly in metropolitan areas, should be prepared to treat radiation expo- sures, because the threat has increased substantially in the past several months.

\section{References}

1. Jarrett DG: Medical Management of Radiological Casualties Handbook. Bethesda, MD: Armed Forces Radiobiology Research Institute, 1999

2. United States Armed Forces: Joint Publication 3-11. Joint Doctrine for Operations in Nuclear, Biological, and Chemical (NBC) Environments. 2000

Manuscript received January 18, 2002.

Accepted in final form February 20, 2002.

The opinions and views expressed in this article are those of the authors and do not reflect the official policy or position of the US Army, US Navy, Department of Defense, or US Government.

Address reprint requests to: Chris J. Neal, M.D., Walter Reed Army Medical Center, 6900 Georgia Avenue, Building 2, 6446, Washington, D.C. 20307-5001 email: kcneal@starpower.net. 